\title{
Partially Folded States of Mutant Ubiquitin in Mild Denaturing Conditions
}

\author{
Soon-Ho Park
Department of Biochemistry and Molecular Biologv, Research Institute of Ural Sciences. College of Dentistrv, Kangntmg-Fonit National Lniversity, Ganghetung 210-702, Korea. E-mail: spark9anukn.ackr
Received April 13, 2009. Accepted May 26, 2009

\begin{abstract}
Confonmational change of ubiquitin variant with valine to alanine mutation at sequence position 26 was studied by varying solvent $\mathrm{pH}$. Fluorescence emission spectra indicated that this variant ubiquitin has some residual structures in acidic and basic solution as compared to denaturant-induced unfolded state. Far-UV circular dichroic spectra indicated that the base-denatured state had more secondary structure than the acid-denatured state. Near-UV circular dichroic spectra indicated that the aromatic side-chains were in the relatively more rigid enviromment in the base-denatured state than those in the acid-denatured state. Although it appears that the more tertiary structure present in the base-denatured state, refolding reactions measured by stopped-flow fluorescence device suggest that both the acid-and base-denatured states oceur before the major folding transition state. I he acid-and base-denatured states are considered to reflect the early event of protein folding process.
\end{abstract}

Key Words: Protein folding. Partially folded state. Folding intermediate

\section{Introduction}

Proteins are polymeric chain molecules composed of aminoacids covalently linked by peptide bond. After sy'nthesized in the ribosome, the emerging chain molecule should be folded into its unique three-dimensional structure to perform biological function. This has been called as the protein folding process. It has been thought that protein folding process occurs from the unstructured random coil state where many possible conformations are in fast equilibrium to the unique native state via intermediate states. These intermediate states are considered to have subsets of the interactions that are present in the native state. ${ }^{\cdot \cdot 4}$ Thus. it has been considered that elucidation of intermediate state conformation is crucial to the understanding of protein folding process. Protein folding kinetics studies indicated the presence of intermediate states which formed transiently in an early stage of folding reaction. ${ }^{5-7}$ However. due to the highly cooperative nature of the protein folding reaction. folding intermediate states are seldom populated at equilibrium for detailed conformational study to understand the protein folding process. Interestingly. it has been observed that the partially denatured, hence partially folded. states are populated at equilibrium when the native protein is in the mild denaturing conditions. ${ }^{4}$ It is thought that the conformation of these partially folded states would provide valuable information for understanding protein folding process.

Ubiquitin. a small globular protein with 76 amino acid residues, has been widely used as a model protein to understand protein folding mechanism. protein stability. and protein design. ${ }^{8}$ However. since the native three-dimensional structure of wild type ubiquitin has been observed to be highly stable and the folding reaction was highly cooperative. partially folded folding intermediate state has not been observed at equilibrium for detailed conformational study. Furthermore. folding kinetics measurements of wild type ubiquitin has been controversial. Khorasanizadeh et al observed the burst phase in the folding kinetics of wild type ubiquitin. ${ }^{9}$ Based on this observation and quantitative kinetic modeling. they argued that wild type ubiquitin folds through at least one intermediate state which is only transiently populated in the early stage of folding. However, this transient intermediate was not observed in other report. ${ }^{101}$ Yet another experiment reported that the burst phase is due to the transient aggregation in the early stage of wild type ubiquitin folding reaction. ${ }^{1]}$ In order to avoid this controversial arguments. we decided to focus on the folding study of nutant ubiquitin with valine 26 to alanine substitution (V26A ubiquitin). since no burst phase was observed in the folding kinetics of this mutant ubiquitin. As shown in Fig. 1. valine 26 is located at the hydrophobic core of wild type ubiquitin. The mutation of valine 26 to alanine expected to remove significant amount of hydrophobic interactions in the core region. Indeed the valine 26 to alanine mutation

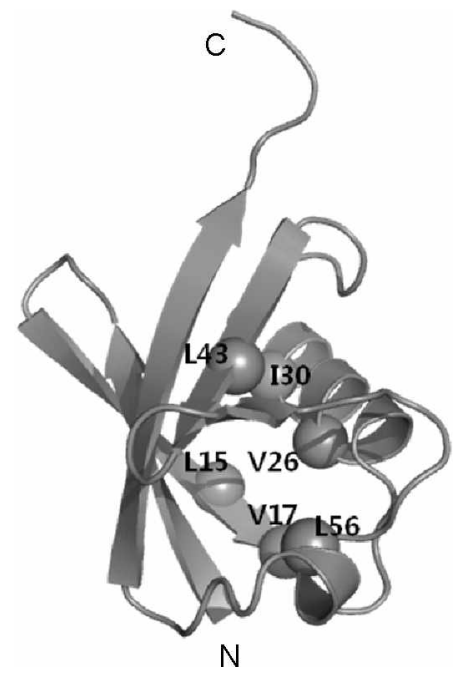

Figure 1. Ribbon diagram of wild type ubiquitin native contonmation. Residues that are contact with valine 26 are shown as spheres. $\mathrm{N}$ and $\mathrm{C}$ denote amino-terminus and carboxy-terminus, respectively. 
destabilizes the native conformation of wild type ubiquitin significantly. ${ }^{9}$ It has been shown that some proteins acquire partially folded conformation in extreme solvent $\mathrm{pH}$." $^{\text {"2 }}$ This is mainly due to destabilizing charge repulsion partially outcompetes the stabilizing hy'drophobic interactions. ${ }^{13}$ These observations suggested that conformationally different partially folded states could be generated in the mild denaturing solvent conditions for V26A ubiquitin with destabilizing hydrophobic core mutation. In this study. we generated the acid- and base-denatured states of V26A ubiquitin by varying solvent $\mathrm{pH}$. These denatured states were subjected to various spectroscopic methods to study conformational properties. Both acid- and base-denatured state of V26A ubiquitin observed to have different conformation than the denaturant-induced unfolded state. The base-denatured state of V26A ubiquitin observed to have more secondary and tertiary structures than the acid-denatured stnicture. Folding kinetics showed that both acid-and base-denatured states occur before the major folding transition state. suggesting that detailed conformational study of these states will expand our understanding of early stage of protein folding.

\section{Materials and Methods}

Materials. Purification of V26A ubiquitin was carried out as previously described method with slight modifications. ${ }^{14}$ Since V26A ubiquitin observed to be less stable than WT ubiquitin, ${ }^{9}$ heating step at $85^{\circ} \mathrm{C}$ for $5 \mathrm{~min}$ was omitted to improve the yield. The Q-sepharose Fast Flow column chromatography was replaced by DEAE-sephacel column chromatography. After DEAE-sephacel column chromatography. V26A ubiquitin was further purified by Sephacryl S-100 column chromatography. The purified V26A ubiquitin was more than $95 \%$ pure judged by the densitometric analysis of coommassie brilliant blue stained gel. The chemical reagents were reagent grade or better

Spectral Measurements. Solutions with various $\mathrm{pH}$ values were generated by adding either $\mathrm{HCl}$ or $\mathrm{NaOH}$ in pure water. Stock solution of V26A ubiquitin was added in each of these solutions and incubated for more than $5 \mathrm{~min}$ at $25^{\circ} \mathrm{C}$ to reach the equilibrium and then the spectrum was collected. The $\mathrm{pH}$ values after spectral measurements were same as the $\mathrm{pH}$ values before the spectral measurements. The unfolded spectrum was measured in the presence of $6 \mathrm{M}$ urea. Intrinsic tryptophan fluorescence emission spectra were collected by using JASCO model FP6500 spectrofluorometer. Tryptophan side-chain was excited at $295 \mathrm{~nm}$ with $5 \mathrm{rm}$ slit width, and the emission spectra from 300 to $450 \mathrm{~nm}$ were measured with the $10 \mathrm{~nm}$ slit width. The protein concentration of fluorescence measurements was $3 \mu \mathrm{M}$. Near- and Far-UV circular dichroic (CD) spectra were measured by using JASCO model J-810 spectroploarimeter. For collecting far-UV CD spectra. about $30 \mu \mathrm{M}$ of protein samples were placed in a quartz cuvette with $1 \mathrm{~mm}$ light path. For collecting near-UV CD spectra. around $50 \mu \mathrm{M}$ of protein samples were placed in the quartz cuvette with $1 \mathrm{~cm}$ light path. For $\mathrm{CD}$ spectra measurements. the band width for far-UV CD spectra measurements was $10 \mathrm{num}$ and that of near- UV CD spectra measurements was $2 \mathrm{~nm}$. All the measurements were made at $25^{\circ} \mathrm{C}$. Reported $\mathrm{CD}$ signals. [ $\theta$ ]. are mean residue ellipticities with the unit of $\mathrm{deg} \mathrm{\textrm {cm } ^ { 2 }} \mathrm{dmol}^{-1}$. For measurements of equilibrium unfolding reaction, fluorescence enussion signal at $355 \mathrm{~nm}$ was monitored in the increasing concentrations of urea after excitation at $295 \mathrm{~nm}$.

Equilibrium Unfolding Measurements. Equilibrium unfolding experiments in the varying solvent $\mathrm{pH}$ and varying urea concentrations were carried out as described by Khorasanizadeh et al. ${ }^{15}$ Urea-dependent unfolding experiments were analyzed assuming a two-state folding transition. $\mathrm{N} \rightleftharpoons \mathrm{U}$. where $\mathrm{N}$ and $\mathrm{U}$ represent the native and unfolded states, respectively. Based on the two-state model, the free energy of unfolding can be calculated by equilibrium constant. $K_{\infty i 1}=$ $[\mathrm{N}] /[\mathrm{U}]$ and $\Delta \mathrm{G}_{\mathrm{U}}=-\mathrm{RT} / n K_{\mathrm{c}, \mathrm{s}}$ where $\Delta \mathrm{G}_{\mathrm{C}}$. R. T represent the free energy' of unfolding. gas constant and absolute temperature. respectively. The free energies of unfolding at each urea concentration was further analyzed by linear extrapolation method as described by equation 1

$$
\Delta \mathrm{G}_{\mathrm{L}}=\Delta \mathrm{G}_{\mathrm{U}^{\prime}}^{\prime \prime}-\mathrm{m}_{\mathrm{eq}} C
$$

where $\Delta \mathrm{G}_{\mathrm{U}}^{\prime \prime}, \mathrm{m}_{\mathrm{eq}}$, and $C$ represent the free energy of unfolding at $0 \mathrm{M}$ urea. slope of the transition curve. and urea concentration. respectively. ${ }^{16}$

Stopjedl-flow Fluorescence Measurements. Refolding kinetics of V26A ubiquitin from various denatured state to the native state was measured by BioLogic SFM- 4 stopped-flow device with fluorescence mode. V26A ubiquitin in $6 \mathrm{M}$ urea solution was diluted $1 \mathrm{l}$-fold with the $25 \mathrm{mM}$ acetate buffer at $\mathrm{pH} 5$ to measure refolding from fully unfolded state to the native state. To measure refolding from acid- and base-denatured state to the native state. acid- and base-denatured V26A ubiquitin was diluted 11 -fold with appropriate buffer to make final $\mathrm{pH}$ of 5 . These measurements were carried out in the presence of $0.55 \mathrm{M}$ urea to make the same final solution condition as refolding reaction from urea-induced unfolded state. The tryptophan side-chain was excited at $295 \mathrm{~cm}$ and the change in fluorescence emission above $324 \mathrm{~nm}$ was measured using a $324 \mathrm{~nm}$ cut-off filter. The fluorescence signals were collected at $1 \mathrm{~ms}$ interval up to $50 \mathrm{~ms}$. $20 \mathrm{~ms}$ interval from 50 ms to $1 \mathrm{sec}$. and $100 \mathrm{~ms}$ interval from $1 \mathrm{sec}$ to $15 \mathrm{sec}$. The dead-time of the stopped-flow device was measured to be 4 ms using the method described by Peterman."

\section{Results and Discussion}

Denaturation of V26A ubiquitin in a mild denaturing condition was explored by measuring intrinsic tryptophan fluorescence emission spectra in the varying solvent $\mathrm{pH}$. As shown in Fig. 2A. intrinsic tryptophan fluorescence emission spectrum of the native state at $\mathrm{pH} 5$ (Fig. 2A solid line) showed the emission maximum at $338 \mathrm{~nm}$. This observation is consistent with the fluorescence emission spectnum of the tryptophan side-chain in the hydrophobic environment of protein interior. ${ }^{18}$ The wavelength of fluorescence emission maximum of $\mathrm{V} 26 \mathrm{~A}$ ubiquitin in solution at $\mathrm{pH} 2$ (Fig. 2A dotted line) was shifted to $351 \mathrm{~nm}$ with increase of the fluorescence emission intensity as compared to that of the 

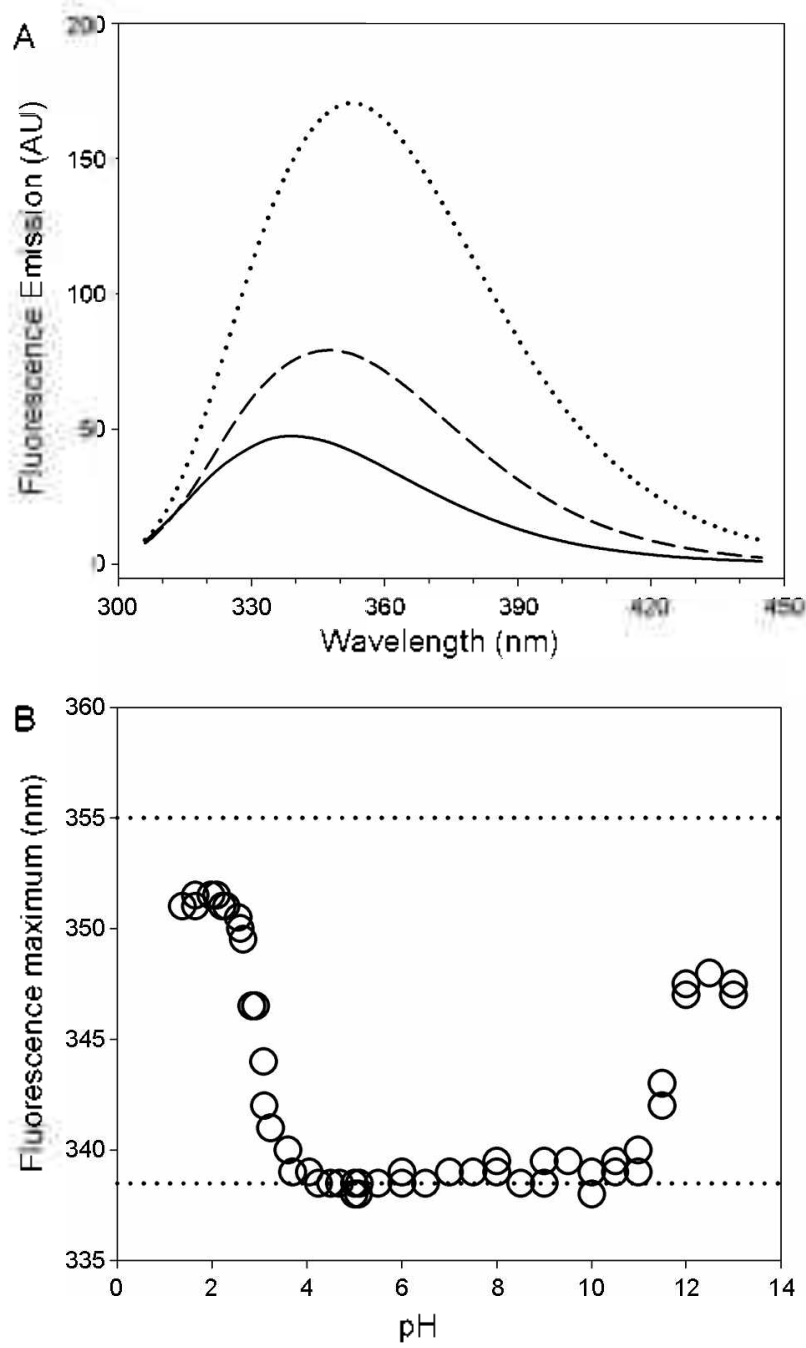

Figure 2. Intrinsic tryptophan fluorescence measurements of V26A ubiquitin. Panel A is the fluorescence enission spectra in solution at pH 2 (dotted line), 5 (solid line), and 12.5 (dashed line). Panel B illustrates the wavelength of fluorescence emission maxima as a function of solvent $\mathrm{pH}$. Upper and lower dotted lines represent the wavelength of fluorescence emission maxinna of fully unfolded state and the native state, respectively. AU denotes arbitrary unit.

native state. The fluorescence emission spectrum at $\mathrm{pH} 12.5$ (Fig. 2A dashed line) showed emission maximum at $347 \mathrm{~nm}$ with modest increase of fluorescence emission intensity as compared to that of the native state. The intrinsic tryptophan fluorescence emission spectrum of fully unfolded ubiquitin in the solution containing high concentration of denaturants has previously been shown to have emission maximum near 355 num with highly increased fluorescence emission intensity as compared to that of the native state. These observations indicate that the native conformation of V26A ubiquitin is denatured in acidic and basic solutions. This suggests that V26A ubiquitin would be useful model to study conformational aspects of denatured state of polypeptide chain. Wild type ubiquitin did not show conformational change in the same solvent conditions. ${ }^{15,19.2(1}$ Fig. $2 \mathrm{~B}$ shows the wavelength of fluorescence emission maximum as a function of solvent $\mathrm{pH}$. Between $\mathrm{pH}+$ and 11. the wavelength of fluorescence emission maximum was observed to be constant at $338 \mathrm{~nm}$.
This spectral feature indicates that little conformational change occurs at these $\mathrm{pH}$ ranges. Red shift of fluorescence emission maximum was observed at the $\mathrm{pH}$ values below 4 and above 11. The wavelength of fluorescence enission maxima appeared to be leveled off at $35 \mathrm{I}$ and $347 \mathrm{~nm}$ in acidic and basic solvent conditions. respectively, suggesting that V26A ubiquitin reached to a certain conformational state at each extreme solvent $\mathrm{pH}$. The wavelength of fluorescence emission maxinta at acidic, $351 \mathrm{~nm}$, and basic, $347 \mathrm{~nm}$, solutions are smaller than that of the fully unfolded state, $355 \mathrm{~mm}$, in solution with high concentration of denaturant. These observations suggest that denatured states of V26A ubiquitin at acidic and basic solutions are different to each other and to the denaturant-induced fully unfolded state. The denatured states in acidic and basic solution appear to be less disordered than that in the high denaturant solution. The wavelength of maxinum fluorescence emission indicates that the side-chain of a single tryptophan residue is in a more hydrophobic environment in a basic solution than that in an acidic solution. The fluorescence emission intensity indicates that the conformation of V26A ubiquitin in basic solution is less denatured than that in acidic solution.

The major force for denaturation of V26A ubiquitin in acidic and basic solutions is considered to be the charge repulsion. V26A ubiquitin has 11 positively charged residues. 7 lysines and 4 arginines. and 11 negatively charged residues, 5 aspartates and 6 glutamates. at neutral $\mathrm{pH}$. Since the $\mathrm{pK}_{\mathrm{c}}$ values for side-chains of aspartate and glutamate are around 4 . it is considered that all the acidic side-chains become neutral at $\mathrm{pH} \mathrm{2}$, so that V26A ubiquitin may have maximum net charge of +11 at the $\mathrm{pH}$ below 2 . By contrast. due to the high $\mathrm{pK}_{\mathrm{a}}$ value of arginine side-chain. which is around 12.5. it is considered that V26A ubiquitin in highly basic solution would have net charge bigger than -11 and has less charge repulsion than in the acidic solution. Thus. it is considered that the stabilizing interactions and the destabilizing charge repulsions are balanced in the more structured denatured state in the basic solution than in the acidic solution for V26A ubiquitin.

The conformational properties of V26A ubiquitin were further explored by CD spectroscopy: The far-UV CD spectnum of the native state V26A ubiquitin (Fig. 3A solid line) shows negative peaks near 222 and $208 \mathrm{~nm}$ with molar ellipticity value of $-4600 \mathrm{deg} \mathrm{cm}^{2} \mathrm{dmol}^{-3}$ at $222 \mathrm{~nm}$, which is consistent with previous observations. ${ }^{21.22}$ The far- UV CD spectrum at acidic pH (Fig. 3A dotted line) appears to be significantly different from that of the native state at $\mathrm{pH} 5$. indicating that the conformation of polypeptide backbone is disnupted. ${ }^{23}$ However, the negative molar ellipticity value at $222 \mathrm{~mm}$ is observed to be similar to that of the native state. suggesting that some secondary structures still remain in this solvent condition. The far-UV CD spectrum of V26A ubiquitin in basic solution observed to have two negative peaks near 222 and $204 \mathrm{lum}$ with the molar ellipticity value of $-5400 \mathrm{deg} \mathrm{\textrm {cm } ^ { 2 }}$ $\mathrm{dmol}^{-1}$ at $222 \mathrm{~mm}$. The movement of $208 \mathrm{~nm}$ peak to $204 \mathrm{~nm}$ with modest decrease of molar ellipticity suggests that the backbone conformation of V26A ubiquitin has been slightly altered upon the addition of base. The peaks at 222 and 204 nn indicate that some helical conformation exists in this solvent condition. Tertiary structures near the amino acid 

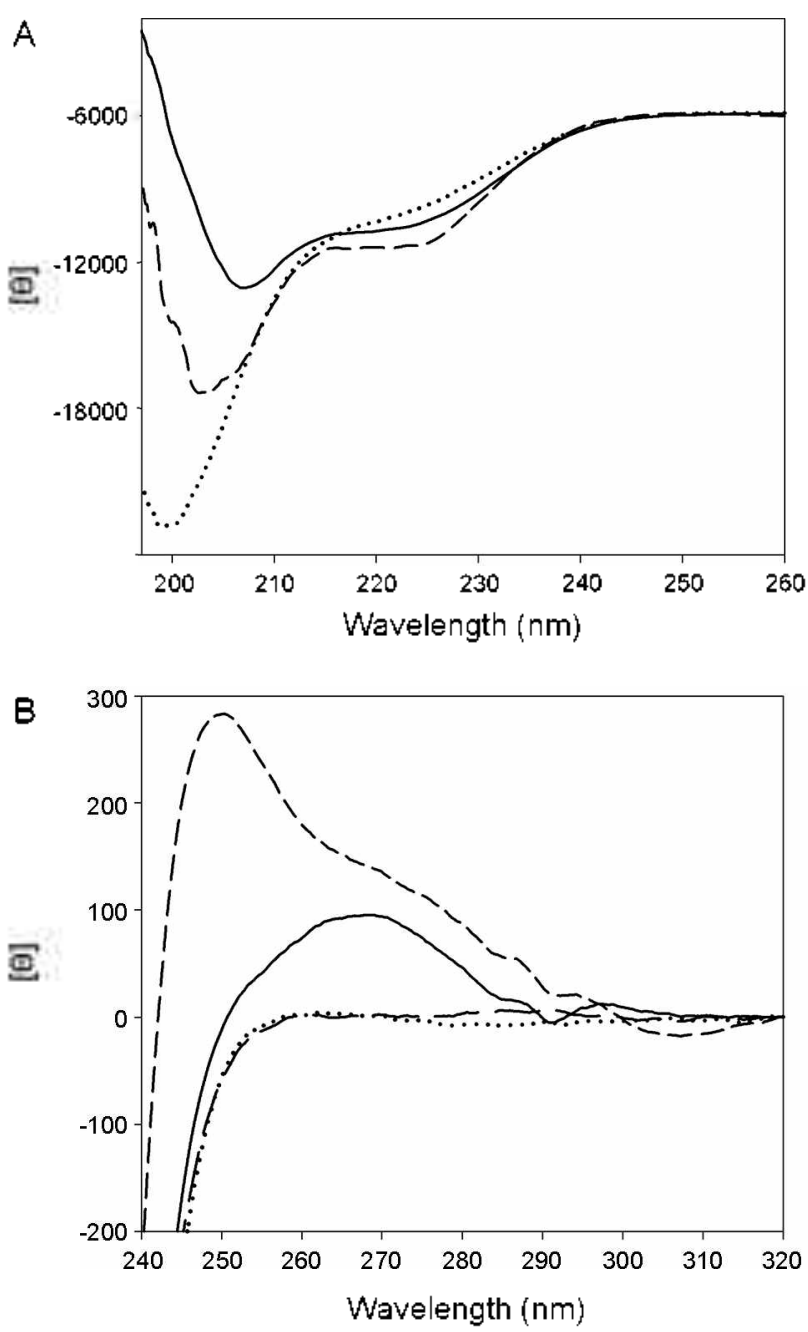

Figure 3. Far- and near-UV CD spectra. Panel A illustrates the far-UV CD spectra of V26A ubiquitin at $\mathrm{pH} 2$ (dotted line), 5 (solid line), and 12.5 (dashed line). Panel B illustrates the near-UV CD spectra. The designations of line in Panel B are sanne as Panel A except long dashed line, which represents the near-UV CD spectrum of unfolded V26A ubiquitin in $6 \mathrm{M}$ urea solution. The molar ellipticity, $[\theta]$, has a unit of deg $\mathrm{cm}^{2} \mathrm{dmol}^{-1}$.

residue with aromatic side-chains of V26A ubiquitin were studied by near-UV CD spectroscopy. Fig. 3B illustrates the near-UV CD spectra of the native (solid line). acid-denatured (dotted line), base- denatured (short dashed line) and fully unfolded (long dashed line) state of V26A ubiquitin. Near-UV spectrum in the native state shows shoulder around $250 \mathrm{~mm}$. broad peak at $270 \mathrm{~mm}$, and small peaks around $290 \mathrm{rum}$. The peaks around $250 \mathrm{~nm} .270 \mathrm{~nm}$. and $290 \mathrm{~nm}$ in the near-UV CD spectra of proteins are considered due to the side-chains of tyrosine tryptophan, and phenylalanine, respectively, when the side-chains of these residues are located in the tightly packed tertiary stnicture. ${ }^{24}$ The near-UV CD spectra of the urea-induced unfolded state and the acid-denatured state show no distinct peaks. indicating that tertiary structures around the side-chains of aromatic residues are disnupted. It is considered that the aromatic side-chains of V26A ubiquitin in acidic solution are located in the hydrophobic cluster to show some fluorescence emission signal but not in the rigid environment

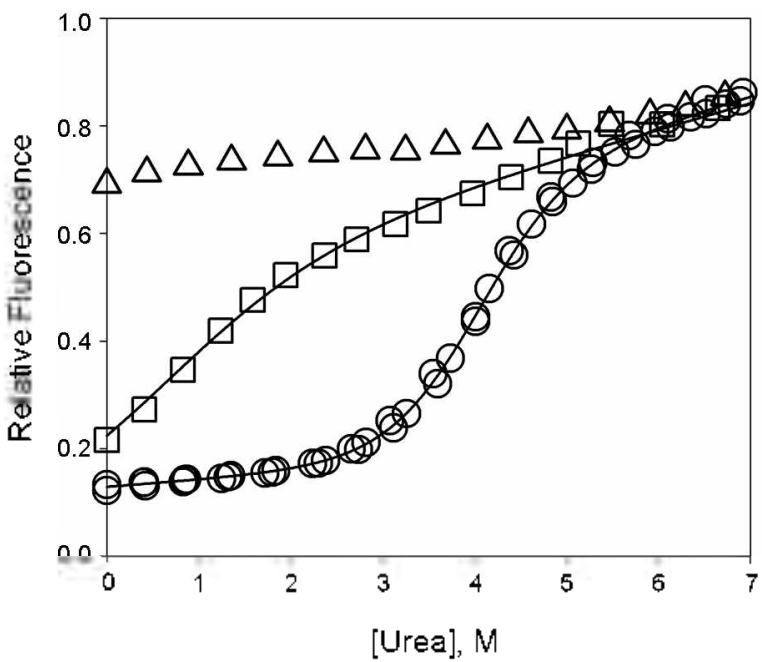

Figure 4. Urea-induced equilibrium unfolding of V26A ubiquitin. Circles, triangles, and squares represent the equilibrium unfolding of $\mathrm{V} 26 \mathrm{~A}$ ubiquitin in the solution at $\mathrm{pH} 5,2$, and 12.5 , respectively. Solid lines denote the nonlinear least squares fit of the untolding data based on a two-state transition model as described in Materials and Method.

to show distinct near-UV signal. Near-UV CD spectrum of base-denatured state of V26A ubiquitin shows peak at 250 $\mathrm{nm}$, shoulder at $270 \mathrm{run}$. and small peaks around $290 \mathrm{lum}$. This spectrum suggests that there are significant amount of tertiary structures exist in the base-denatured state of V26A ubiquitin. The peak at $250 \mathrm{~mm}$ in the base-denatured V26A ubiquitin suggests that the side-chain of a single ty rosine residue has somewhat different orientation than that in the native state

The equilibrium unfolding transitions of V26A ubiquitin at $\mathrm{pH} 2$ (triangle), 5 (circle), and 12.5 (square) are illustrated in Fig. 4. The unfolding transition of V26A ubiquitin at $\mathrm{pH} 5$ shows typical cooperative transition pattern of sigmoidal shape. indicating that V26A ubiquitin has tightly packed hydrophobic core. ${ }^{35}$ This transition curve was fitted to the linear extrapolation method (LEM) with $\Delta \mathrm{G}^{\circ}{ }^{\circ}$ and $\mathrm{ml}_{\mathrm{e}_{\mathrm{q}}}$ of 4.65 $\mathrm{kcal} \mathrm{mol}{ }^{-1}$ and $1.16 \mathrm{kcal} \mathrm{mol}^{-1} \mathrm{M}^{-1}$. respectively: No cooperative sigmoidal transition was observed at $\mathrm{pH} 2$. suggesting that no tightly packed hydrophobic core exists in V26A ubiquitin at this solvent $\mathrm{pH}$. This observation is consistent with the near-UV CD spectrum. The fluorescence signal increases monotonously as increasing urea concentrations at $\mathrm{pH} 12.5$, suggesting less cooperative conformational transition for V26A ubiquitin at this solvent $\mathrm{pH}$. This observation suggests that denatured state of V26A ubiquitin at $\mathrm{pH} 12.5$ has some interactions in the hydrophobic core to show shallow cooperative folding transition. Although the baseline of the native state was not clearly observed. the transition curve at $\mathrm{pH} 12.5$ was fitted to LEM assuming the same native baseline as the transition at $\mathrm{pH} 5$. The $\Delta \mathrm{G}^{\circ}{ }^{\circ}$ and $\mathrm{m}_{\mathrm{ej}}$ were fitted to 0.28 $\mathrm{kcal} \mathrm{mol}{ }^{-1}$ and $0.64 \mathrm{kcal} \mathrm{mol}^{-1} \mathrm{M}^{-1}$, respectively. Based on the $\Delta \mathrm{G}_{\mathrm{O}}^{\circ}$, the conformational stability of V26A ubiquitin is decreased by about $90 \%$. indicating that the conformation fonmed at $\mathrm{pH} 12.5$ is only marginally stable. The $\mathrm{m}_{\text {eq }}$ value has been interpreted to represent the solvent accessible surface area upon the unfolding of the native proteins. ${ }^{55}$ The bigger 


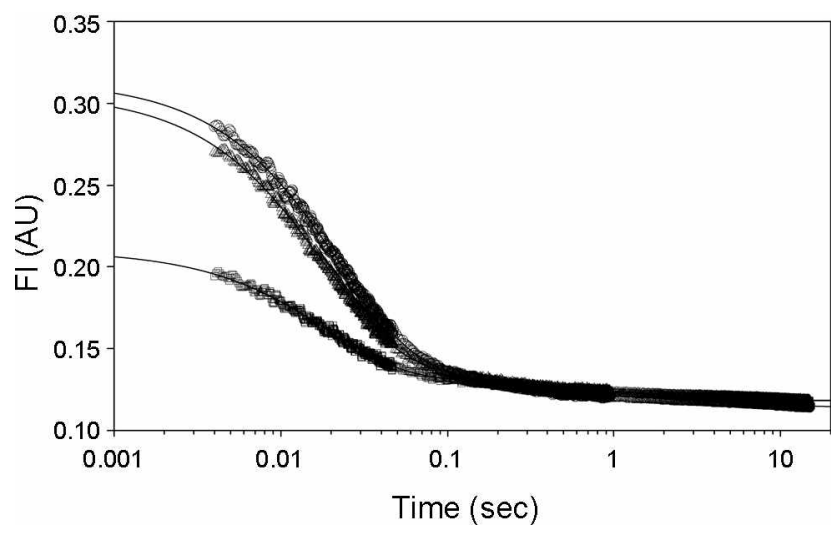

Figure 5. Kinetic traces of refolding of V26A ubiquitin. Circles, squares, and triangles represent the fluorescence emission traces of refolding for acid-denatured state at $\mathrm{pH} 2$, base-denatured state at $\mathrm{pH}$ 12.5. and unfolded state in $6 \mathrm{M}$ urea. respectively. Solid lines are obtained by nonlinear least squares analysis of the folding kinetic traces with three exponentials.

$m_{e q}$ value suggests that the more surface area is exposed upon unfolding. The obtained $m_{\text {eq }}$ value of the base-denatured $V 26 \mathrm{~A}$ ubiquitin is about $5 \mathbf{5} \%$ of that of the native state. Since the solvent accessible surface area would be completely exposed in unfolded state at high concentration of urea. it is considered that about $45 \%$ of solvent accessible surface area is already exposed at $0 \mathrm{M}$ urea for V26A ubiquitin in the solution at $\mathrm{pH}$ 12.5. This observation together with near-UV CD spectrum suggests that the conformation of V26A ubiquitin at $\mathrm{pH} 12.5$ is significantly collapsed hydrophobic core. which provides some asy mmetric environment to the aromatic side- chains. It has been observed that about $35 \%$ of the solvent accessible surface area was exposed in the folding transition state of ubiquitin. ${ }^{9}$ Thus it is considered that. although there are significant amount of tertiary structures in the base-denatured state, the base-denatured state is less compact than the folding transition state. suggesting that the based-denatured state might occur before the major folding transition state in the V26A ubiquitin folding reaction.

Refolding reactions from acid- and base-denatured state to the native state were measured together with refolding reaction from denaturant-induced unfolded state to the native state by using stopped-flow fluorescence device. In this experiment. each denatured state was rapidly mixed with refolding buffers to give a same final solution so that the folding kinetics can be followed in the same solvent condition. As shown in Fig. 5. the kinetic traces show typical exponential change in fluorescence emission upon the initiation of refolding. The initial fluorescence emission signal for the refolding reaction of base-denatured state appears to be closer to the fluorescence signal of the native state than those for acid-denatured and denaturant-induced unfolded state. This obserwation suggests that base-denatured state is more stnictured than the acid-denatured and denaturant-induced unfolded state at the initial folding time. Each refolding kinetic trace was fitted with three exponentials consistent with previous observations. ${ }^{9.15}$ The fitting parameters of these kinetic traces are listed in Table 1. It has been interpreted previously that two slower phases.
Table 1. Analy sis of folding kinetics of $\mathrm{V} 26 \mathrm{~A}$ ubiquitin from various denatured states.

\begin{tabular}{lcccccc}
\hline Denaturant & $\lambda_{1}$ & $A_{1}$ & $\lambda_{2}$ & $A_{2}$ & $\lambda_{3}$ & $A_{3}$ \\
\hline Acid $(\mathrm{pH} \mathrm{2})$ & $45.2 \pm 1.1$ & 85 & $6.0 \pm 0.5$ & 11 & $0.18 \pm 0.01$ & 4 \\
Base (pH 12.5) & $54.3 \pm 2.1$ & 79 & $5.5 \pm 0.5$ & 13 & $0.25 \pm 0.02$ & 8 \\
Urea & $46.7 \pm 1.1$ & 87 & $4.4 \pm 0.3$ & 8 & $0.10 \pm 0.01$ & 5 \\
\hline
\end{tabular}

The unit for rate constant $(\omega)$ is $\mathrm{sec}^{-1}$. Amplitude (A) is expressed as percent. Errors of amplitudes were less than $3^{0} \cdot$. Subscripts 1. 2, and 3 represent the first, second, and the third kinetic phases. respectively.

about $15 \%$ of total anplitude. are due to the parallel folding process caused by the configurational isomenization of peptide bond. ${ }^{y}$ The first exponential phase, about $85 \%$ of total anplitude. was considered to reflect the confonnational folding process in the ubiquitin folding kinetics. ${ }^{9}$ The folding rate for base-denatured state to the native state is slightly faster than those for the acid-denatured and denaturant-induced unfolded state to the native state. The folding rates for acid-denatured and unfolded state to the native state were observed to be comparable. These results suggest that the base-denatured state would be closer to the native state than the acid-denatured and denaturant-induced unfolded states in the folding reaction coordinate. This is consistent with the spectroscopic and equilibrium unfolding measurements that the base-denatured state appears to be more compact and have more secondary and tertiary structures than the acid-denatured and the denaturant-induced unfolded state. However observation of major folding phase in refolding kinetics of base-denatured state to the native state suggests that the base-denatured state occurs before the major folding transition state, which is consistent with the interpretation of urea-induced unfolding experiment. If base-denatured state occurs after major folding transition state, the first folding phase should not be observed. Therefore. although base-denatured state is observed to have significant amount of secondary and tertiary structures, it is considered less structured than the major folding transition state. Furthermore, the observation that folding rates for acidand base-denatured states are faster or conparable to the folding rate for unfolded state suggest that acid- and base-denatured states would be on-pathway in V26A ubiquitin folding process. If these states are not on the folding pathway. the rates of folding should be significantly slowed since off-pathway states should be unfolded before the refolding reaction proceeds. It is considered that the side-chain orientation of the tyrosine residue at $\mathrm{pH} 12.5$ that is shown to be different from the native state would not linder the folding process of V26A ubiquitin.

The detailed conformational study of folding intermediate state was fairly difficult in the solvent condition that favors the native conformation. since an intermediate state of folding reaction is seldom populated due to the highly cooperative nature of protein folding reaction. The denatured states observed in the mild denaturing condition in this study suggests that detailed confonnational properties of polypeptide chain in the middle of the folding process can be studied at equilibrium and shed light on the understanding of protein folding process. 


\section{Concluding Remarks}

Denaturation of V26A ubiquitin was stıdied by varying the solvent $\mathrm{pH}$. V26A ubiquitin started to denature at the $\mathrm{pH}$ values below 4 and above 11 and reached a certain denatured state at the $\mathrm{pH}$ values below 2 and above 12 . respectively. The acid- and base-denatured states were observed to be conformationally different. The near-and far-UV CD measurements indicated that the base-denatured state appeared to have some secondary and tertiary conformations. The acid-denatured state appeared to have some secondary structures but no tightly packed hydrophobic core. Equilibrium unfolding measurement indicated that the base-denatured state appeared to have partially collapsed hydrophobic core. Folding kinetics measurements indicated that both the acid- and the base- denatured states are on the folding pathway. Furthermore, these conformational states appear to occur before the major folding transition state. All these observations clearly indicate the possibility of generating intermediate states with varying amount of secondary and tertiary structures in V26A ubiquitin so that we can further study the conformational properties of states occur on the protein folding pathway: It is considered that the elucidation of the conformation of acid- and basedenatured states of V26A ubiquitin at the residue level would be very informative to understand the early stage of protein folding process.

Aclnowledgments. Author appreciates to Dr. Myeong-Hee $\mathrm{Yu}$ and Dr. Cheolju Lee in Korea Institute of Science and Technology for providing stopped-flow device. This work was supported by the grant from Kangnung-Wonju National University

\section{References}

1. Kim, P. S.; Baldwin, R. L. Anmt. Rev Biochent. 1982, 51, 459.
2. Kim. P. S.; Baldwin. R. L. Amm. Rev. Biochem. 1990, 59, 631.

3. Matthews, C. R. Amm. Rev Biochem. 1993, 62, 653.

4. Ptitsyn, O. B. Ads Protein Chem. 1995, 47,83 .

5. Kuwạima, K.: Hiraoka, Y.: Ikeguchi, M.: Sugai, S. Biochemistr y $1985,24,874$.

6. Arai, M.; Kuwajima, K. Fold Des 1996, 1, 275.

7. Park, S.-H.: O'Neil, K. T.: Roder, H. Biochemism 1997, 36, 14277 .

8. Jackson, S. E. Oig. Biomol. Chem. 2006, $4,1845$.

9. Khorasanizadeh, S.; Peters, I. D.; Roder, H. Kat. Sintct. Biol. $1996,3,193$.

10. Krantz, B. A.; Sosnick, T. R. Biochemistry 2000, 39, 11696.

11. Went, H. M.; Benitez-Cordoza, C. G.; Tackson, S. E. FEBS Lett. 2004, 567,333 .

12. Fink, A. L.; Calciano, L. .T.; Goto, Y,; Kurotsu, T.; Palleros, D. R. Biochemisty 1994, 33, 12504.

13. Uversky, V. N. FEBS Lett. 2002, 514, 181 .

14. Ecker, D. J.; Khan, M. I.; Marsh, T; Butt, T. R.; Crooke, S. T. J. Biol. Chem. 1987, 262,3524.

15. Khorasatizadeh, S.; Peters, I. D.; Butt, T. R.; Roder, H. Biochentistry $1993,32,7054$

16. Pace, C. N. CRC Crit. Rev. Biochem. 1975, 3, 1.

17. Peterman, B. F. Anal. Biochem. 1979, 93, 442.

18. Stryer, L. Science 1968, 162, 526.

19. Wintrode, P. L.; Makhatadze, G. I.; Privalov, P. L. Proteins 1994, $18,246$.

20. Park, S.-H. J. Biochem. Mol. Biol. 2004, 37,676.

21. Wilkinson, K. D.; Mayer, A. N. Arch. Biochem. Bioplys. 1986, 250,390

22. Cox, J. P. L.; Evans, P. A.; Packman, L. C.; Williams, D. H.; Woolfson, D. N. J. Hot. Biol. 1993, 234, 483.

23. Greenfield, N.: Fasman, G. D. Biochemistiv 1969, 8, 4108.

24. Sears, D. W; Beychok, S. Cincular Dichroism, in Phwical Principles and Techniques of Protein Chemishy Pan C; Leach, S. J., Ed.: Academic Press: New York, U.S.A., 1979; p 446.

25. Myers, J. K.; Pace, C. N.; Scholtz, J. M. Protein Sci. 1995, 4, 2138.

26. Crespo, M. D; Platt, G. W; Botill, R.; Searle, M. S. Eur: J. Biochem. 2004, 271, 4474 . 